\title{
LAS REFORMAS DE LA EDUCACIÓN SUPERIOR EN ARGENTINA PARA EL NUEVO MILENIO
}

\author{
Marcela Mollis*
}

\begin{abstract}
* Profesora de Historia de la Educación y Educación Comparada. Directora del Programa de Investigaciones en Educación Superior Comparada, IICE, de la Universidad de Buenos Aires. Miembro del Foro UNESCO de educación superior, investigación y conocimiento. Miembro del Grupo de Trabajo de CLACSO sobre Universidad y Sociedad. Autora de numerosos libros, capítulos de libros y artículos sobre educación superior latinoamericana, entre ellos: Las Universidades en América Latina. CLACSO, 2003. Email: marmollis@, gmail.com
\end{abstract}

Resumen: La autora ofrece un panorama de las raíces del fenómeno de distorsión con relación a su misión que se observa en las universidades argentinas y su deserción de su función social. Sin embargo, Argentina tiene uno de los mejores Sistemas de ES de América Latina y el Caribe en cuanto a nivel académico y una de las tasas más altas de matrícula (el 54\%) en la región.

Palabras claves: Educación Superior. Argentina. Reformas de la ES argentina. Globalización y cultura empresarial. Tendencias y desafíos.

Abstract: The author presents an overview of the roots of the distortion of the mission of Argentinean universities and of their desertion from its social function. Nonetheless, in terms of quality, Argentina has one of the best higher education systems in Latin America and the Caribbean and also the highest enrollment rates (54\%) in the region.

Keywords: Higher education. Argentina. Reforms of Argentinean higher education. Globalization and business culture. Trends and challenges.

\section{Introducción}

\section{El escenario de las reformas de la educación superior en la Argentina}

Desde 1995 en adelante, en Argentina -como en México, Brasil, Bolivia, Rusia, Bulgaria y Mongolia, entre otros países de América Latina y del ex bloque socialista-, se instaló una "agenda internacional de la modernización de los sistemas educativos superiores" promovida fundamentalmente por las agencias de crédito internacional como el Banco Mundial y el Banco Interamericano de Desarrollo (Marginson, S. \& Mollis, M., 2001). Esta agenda se propuso, fundamentalmente, la disminución de los subsidios estatales para la educación y la ciencia y el control selectivo del estado en la distribución de los recursos financieros, la expansión de las instituciones y de la matrícula privadas, la promulgación de una Ley de Educación Superior con consecuencias 
para el sistema de evaluación y acreditación y por último, la creación de órganos o agentes centrales para evaluar y acreditar las instituciones universitarias (como la Secretaría de Políticas Universitarias actualmente llamada Secretaria de Educación Superior y la Comisión Nacional de Evaluación y Acreditación Universitaria -CONEAU-) (Mollis, M., 2001)

a) Disminución de subsidios estatales para la educación, la ciencia y la cultura

La Argentina es uno de los países dentro de una muestra de la OCDE, que menos inversión realiza en la educación superior y en la educación universitaria. El porcentaje que invierte con respecto al Producto Bruto Interno es menor que otros países de América Latina con menor ingreso per cápita como Chile, Brasil, Venezuela y México. El promedio de la mencionada muestra para la educación superior es del 1,3\% del PBI, en cambio Argentina invierte el 0,95\% del PBI. A su vez, el sector privado en nuestro país a diferencia de los países industrializados, prácticamente no tiene participación en el gasto de la educación superior.

Susana Torrado (2001), en un provocativo artículo, afirma que la discusión sobre cómo racionalizar el gasto para la educación superior es falaz. Tanto quienes están a favor como en contra de restringir el ingreso o arancelar, olvidan que las estrategias educativas están diseñadas por el proyecto económico elaborado desde el Ministerio de Economía. Los datos que aporta la investigadora, son fundamentales para comprender que el problema de la diversidad de fuentes de financiamiento no resuelve la reproducción social universitaria, o mejor dicho el auto reclutamiento que la universidad viene realizando desde que los inmigrantes se convirtieron en segunda generación de argentinos. A la pregunta ¿quiénes acceden a la educación? -es decir la probabilidad individual de acceder o terminar diversos niveles educativos según pertenencia a un determinado estrato social- Torrado brinda respuestas aparentemente obvias, con importantes consecuencias para desmitificar el problema del financiamiento de la educación superior. La universidad argentina se auto recluta, es decir, acceden mayoritariamente los hijos de los profesionales que, a su vez, tienen mayores probabilidades de graduarse. Cambiar el sistema de financiamiento alternativo al estatal, no resuelve el problema de los beneficiarios de las universidades, la mayor responsabilidad en la selección social al interior del sistema educativo le corresponde al plan económico que deja fuera del circuito formal a los argentinos más pobres. 
En cuanto a la inversión en investigación y desarrollo, según un informe internacional recientemente elaborado por la Red de Indicadores de Ciencia y Tecnología Iberoamericana (2000), la Argentina invierte menos fondos $(0,24 \%$ del PBI) que en la década del $80(0,37 \%$ del PBI), en menor proporción que los vecinos Chile y Brasil y su inversión es 189 veces menor a la de Estados Unidos. A su vez toda América Latina invierte una cifra total en Investigación y Desarrollo menor a la de Canadá. Para los realizadores de este informe, el esfuerzo del sector público y privado en el área del desarrollo científico y tecnológico de la Argentina es insuficiente y poco sostenido

Aún quienes defienden la tesis modernizadora que el futuro solo es viable para las "sociedades del conocimiento", consideran que estos indicadores representan el abandono del estado y del sector privado de las actividades vinculadas a la investigación básica y aplicada. La desinversión universitaria forma parte del mandato globalizador por el cual se deja la producción del conocimiento innovador en manos de los países altamente industrializados, es decir el desarrollo de la investigación a las universidades y empresas del norte. En el reparto de las funciones mundiales del conocimiento, a nuestras universidades les toca el papel de entrenadoras de recursos humanos, que seguirán siendo humanos en tanto sean "recursos" y no por los planes de estudio humanistas. Finalmente, así se hace realidad la sagaz frase de Britós:

"el lugar que una sociedad le asigna a la universidad, misteriosamente coincide con el lugar que esa sociedad tiene en el mundo"

\section{b) Expansión de las instituciones y la matrícula privadas}

El proceso de privatizaciones, se ha convertido en uno de los dispositivos prioritario para el cambio de régimen de acumulación capitalista en Argentina y en el resto de América Latina. A partir de ese proceso se modifica, en parte, la intervención del Estado en la economía: se abandona la función "reguladora" de las empresas del Estado porque todas se privatizan (electricidad, teléfonos, compañía aérea, yacimientos de petróleo, etc). La cultura está subordinada a las reglas del mercado, el Estado reducido a un conjunto de medidas gubernamentales que muestran ser eficiente a la luz de los intereses de la bolsa de valores

Desde la perspectiva de los hacedores de políticas educativas, la explosión de la demanda por la educación universitaria en el último quinquenio del siglo, promovió una ley (la 24.521) y varios proyectos para reorientar la 
demanda hacia otras instituciones terciarias no universitarias públicas o privadas así como propuestas tendientes al cobro de impuestos directos a las familias de estudiantes universitarios. La explosión de los pos grados (promovidos en parte por la Ley de Educación Superior) refleja la demanda por una mayor capacitación o especialización al terminar el ciclo universitario, y en todos los casos, este tipo de oferta es arancelada.

c) La Ley de Educación Superior No 24.521, fue promulgada el 7 de Agosto de 1995, con gran disenso de parte del movimiento estudiantil, de rectores y profesores universitarios, e incluso de parte de algunos representantes del poder legislativo. La Ley comprende a las instituciones de formación superior, universitarias y no universitarias, nacionales, provinciales o municipales, tanto estatales como privadas, todas las cuales forman parte del Sistema Educativo Nacional. Consta de IV Títulos, subdivididos en Capítulos y Secciones con un total de 89 Artículos. Introduce cambios sustantivos en lo que respecta a los históricos conceptos de autonomía, financiamiento y gobierno universitario. A modo de ejemplo, autoriza a las instituciones universitarias a establecer el régimen de acceso, permanencia y egreso de sus estudiantes en forma autónoma (en las universidades con más de 50.000 estudiantes, el régimen de admisión, permanencia y promoción puede ser definido por cada facultad); autoriza a cada universidad a que fije su propio régimen salarial docente y de administración de personal, asegurándoles el manejo descentralizado de los fondos que ellas generan; pueden promover la constitución de "sociedades, fundaciones u otras formas de asociación civil" destinadas a apoyar la gestión financiera y a facilitar las relaciones de las universidades y /o facultades con el medio; los órganos colegiados tienen funciones de definición de políticas y de control, en tanto que los unipersonales tienen funciones ejecutivas; modifica la integración del claustro de profesores autorizando al conjunto de los docentes (auxiliares inclusive) para ser elegidos como representantes de dicho claustro; y finalmente, aumenta el número de cuerpos representados en los órganos colegiados. Esta extensa ley en su espíritu, es muy diferente a la tradicional ley Avellaneda que rigió los destinos de nuestras universidades por más de 70 años. En los acuerdos producidos para la aprobación de ley, se reconocen intereses corporativos y partidarios que no están intrínsecamente vinculados a la anhelada excelencia para la educación superior. 
d) Creación de la SPU/ SES y de la CONEAU

En Argentina, se produjo un cambio significativo en las políticas dirigidas al nivel superior educativo, a partir de la creación de la Secretaría de Políticas Universitarias en 1994 y la promulgación de la mencionada Ley No 24.521 descripta más arriba.

En 1995 la Comisión Nacional de Acreditación de Pos grados (CAP) puso en marcha un proceso de acreditación de carreras de pos grado en el que se evaluaron, acreditaron y calificaron 176 carreras de pos grado de universidades públicas y privadas. La política de evaluación y acreditación de instituciones y carreras universitarias y de pos grado, formaba parte de la "política de promoción de la calidad" impulsada por el Ministerio de Cultura y Educación. Otros dos programas que contribuyeron en este sentido fueron:

- el Programa de Incentivo a los Docentes-Investigadores, que otorga fondos adicionales para premiar el mayor rendimiento del trabajo académico de los mismos, y

- el fondo para el Mejoramiento de la Calidad Universitaria (F.O.M.E.C.) que, desde 1995, destina fondos a través de concursos para apoyar financieramente procesos de reforma y mejoramiento de la calidad de las universidades nacionales

El mayor impacto lo tuvo la introducción de mecanismos centralizados para la evaluación y acreditación universitaria en manos de la Comisión Nacional de Evaluación y Acreditación Universitaria (CONEAU). Dicha comisión, tiene mandato legal para realizar evaluaciones externas, acreditar carreras de grado y de pos grado, evaluar proyectos institucionales para la creación de nuevas instituciones universitarias nacionales o provinciales, y evaluar el desarrollo de los proyectos para el posterior reconocimiento de instituciones universitarias privadas de parte del Ministerio de Cultura y Educación. La Ley determina que la CONEAU es un organismo descentralizado dentro de la jurisdicción del Ministerio de Cultura y Educación, integrada por doce miembros designados por el Poder Ejecutivo Nacional a propuesta de los siguientes organismos: 3 por el Consejo Inter Universitario Nacional (CIN), 1 por el Consejo de Rectores de Universidades Privadas (CRUP), 1 por la Academia Nacional de Educación, 3 por la Cámara de Diputados de la Nación, 3 por la Cámara de Senadores de la Nación, 1 por el Ministerio de Cultura y Educación de la Nación.

Estas reformas se produjeron en el contexto de las políticas públicas de corte neo liberal que sustentaron la desregulación orientada por la libertad de 
los mercados con su correlato en la libertad otorgada a las universidades para cobrar aranceles, pagar salarios diferenciados y diseñar regímenes de admisión (examen de ingreso, ciclos introductorios, cupos, etc.) junto a la tendencia del gobierno a controlar y "evaluar" el desempeño de las instituciones universitarias (Mollis, M. 2006b). Darle libertad a las instituciones para buscar otras fuentes de financiamiento alternativo al estatal y a su vez, controlar sus resultados a través de órganos de evaluación y acreditación como la CONEAU.

\section{1) La educación superior: dos subsistemas desarticulados}

La educación superior en la Argentina es un caso típico y también específico de un sistema binario, cuya especificidad radica en que la mayor oferta educativa del nivel superior no universitario se concentra en la formación docente comparada con la tendencia internacional de los sistemas binarios (Dono Rubio, S., 2001). A su vez, la mayor concentración de la matrícula se produce en el nivel universitario público. En este sentido se caracteriza por una gran diversidad en su oferta educativa, con significativas superposiciones en cuanto a los títulos y diplomas ofrecidos en el nivel universitario y no universitario y una fragmentación visible en el conjunto del sistema conformado por diferentes tipos de instituciones desarticuladas entre sí. Esta situación se produce como consecuencia de históricas políticas educativas fragmentadas e implementadas por distintos gobiernos, en función de intereses, proyectos políticos y modelos económicos diferentes para la educación. Como consecuencia, el nivel superior de educación ofrece una variedad de instituciones que difieren en la calidad y en sus misiones específicas. Así se desarrolló una fragmentación costosa y conspirativa contra el funcionamiento eficiente del conjunto del nivel superior de educación debido a que los sectores que lo conforman nunca establecieron canales de articulación entre los distintos tipos de formación que el nivel superior brindaba (universitaria y no universitaria) y nunca se reciclaron las ofertas educativas ya instaladas (Dirie, C. Mollis M. y otros, 2001). Por el contrario, las políticas de la educación superior en cuanto a su modalidad de oferta, se puede caracterizar como una superposición fragmentada costosa e ineficiente orientada en la última década por el perfil del potencial consumidor. De acuerdo a los mapas de la oferta consultados, se puede observar el crecimiento acelerado de la nueva oferta pública y privada en las zonas urbanas con mayor densidad poblacional y mayor poder adquisitivo, junto al crecimiento de la oferta privada en las zonas urbanas de las regiones con mayor potencial económico o derivado del desarrollo político provincial. Concomitantemente, el peso de la matrícula 
de estudios superiores (universitarios y no universitarios) en la población total es cada vez mayor. El número de estudiantes por cada 10.000 habitantes pasa de 7 en 1914, a 32.4 en 1950, 106.7 en 1970 y 149 en 1980.

De acuerdo con los datos del Anuario 2005 de Estadísticas Universitarias publicado por la Secretaria de Políticas Universitarias (SPU) en base a la estimación de población entre 18 y 24 años, la tasa neta universitaria es 18, 3\%, la tasa bruta es 33,8\% y la tasa bruta de educación superior es $45 \%$. No obstante la gran expansión y el crecimiento de la cobertura de la educación superior en Argentina en las últimas décadas, su distribución y características distan de ser homogéneas en las distintas provincias y regiones.

\subsection{1) Problemas que surgen por la superposición y desarticulación de la oferta}

- El principio organizacional básico de los sistemas de educación superior diversos - como el norteamericano, el chileno o el finlandés-, es que funcionan como un "todo articulado". Nuestro conjunto de instituciones universitarias y no universitarias, no funciona como un "sistema" con partes articuladas entre sí. El problema entonces no es la "diversidad" de ingresos universitarios y de oferta, sino la falta de planeamiento conjunto y la falta de articulación entre el nivel secundario y el pos secundario.

- El problema de la educación superior argentina no es la masividad en sí, sino la falta de una estructura adecuada a la demanda social creciente por la educación superior. Por eso es indispensable configurar un sistema de educación superior articulado con el secundario que ofrezca además, diversas "rutas" de formación, comunicadas entre sí.

- Si se ofrecieran distintas "rutas de formación" pos secundaria a los jóvenes en edad correspondiente, el porcentaje de egresados universitarios respecto de los ingresantes al sistema, probablemente se elevaría (como sucede en los países industriales) en la medida que los niveles de retención de la matrícula aumentarían en otros segmentos de la educación pos secundaria 
- La coexistencia de diferentes sistemas de ingreso en las universidades obedece a que la educación superior argentina en su conjunto, no es homogénea sino heterogénea. Existe una correspondencia entre la heterogeneidad institucional y la heterogeneidad de los sistemas de ingreso. Lo que es bueno para una institución con determinadas características, puede no serlo para otra. En general los países que tienen sistemas de educación superior con instituciones diversas, tienen diversas políticas de ingreso (Dirié, C. Mollis, M. y Otros, 2001, pág.)

\section{La educación superior argentina en cifras}

El mal llamado "sistema" de educación superior en la Argentina constituye un conglomerado institucional complejo y heterogéneo, conformado por más de 1,700 establecimientos de nivel terciario no universitario y por 102 establecimientos universitarios.

El sistema de educación no universitario está compuesto por institutos de formación docente para los distintos niveles de enseñanza e institutos o escuelas especializadas de orientación técnica. Las instituciones públicas dependen en su gestión y financiamiento de los gobiernos provinciales o de la Municipalidad de la ciudad de Buenos Aires. Por su parte las instituciones privadas se financian por el cobro de aranceles, aunque los establecimientos de formación docente privados reciben también subsidio público cuando los aranceles que cobran son reducidos.

Las 101 instituciones universitarias oficialmente reconocidas en el país hasta el año 2007 están integradas por 38 universidades nacionales, 41 universidades privadas, 6 institutos universitarios nacionales creados bajo el régimen legal de universidades provinciales y 14 institutos universitarios privados, 1 universidad provincial, 1 universidad extranjera y 1 internacional. Todas configuran un mapa cuyos rasgos más característicos son la complejidad, la diversidad, $\mathbf{y}$ una heterogénea multifuncionalidad (esto significa que la misma universidad lleva a cabo múltiples misiones o funciones, como por ejemplo, enseñar, entrenar profesionales, investigar, desarrollar la cultura local y vender servicios).

De acuerdo con el Cuadro 1, podemos reconocer las tendencias a la expansión institucional y a la privatización que predominaron en la década del 90 , por el número de instituciones nacionales y privadas que fueron creadas en dicho periodo. En los últimos dieciséis años se crearon el $24 \%$ del total de las universidades nacionales, el $44 \%$ de las universidades privadas, el $83 \%$ de 
los institutos universitarios nacionales y el 93\% de los institutos universitarios privados, así como el 100\% de las universidades provinciales y extranjeras.

\begin{tabular}{|c|c|c|c|c|c|c|c|c|c|}
\hline \multirow{9}{*}{ 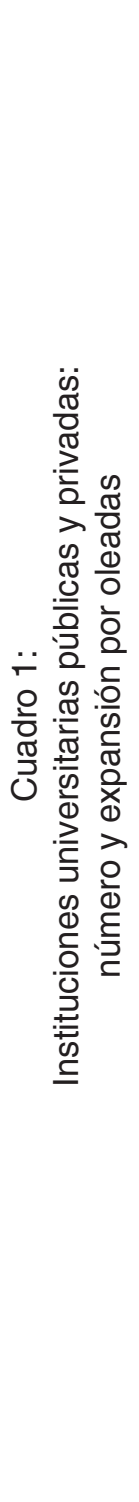 } & \multirow{2}{*}{ 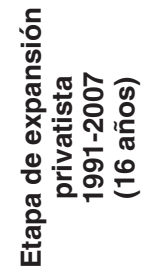 } & $\circ^{\circ}$ & $\widehat{\stackrel{\text { }}{\tilde{N}}}$ & $\begin{array}{l}\stackrel{g}{g} \\
\text { gे }\end{array}$ & $\stackrel{m}{\mathscr{\infty}}$ & $\begin{array}{l}\infty \\
\tilde{\sigma}\end{array}$ & $\stackrel{\circ}{\circ}$ & $\stackrel{\circ}{\circ}$ & \\
\hline & & $z$ & $\sigma$ & $\stackrel{\infty}{\longrightarrow}$ & م & $\stackrel{m}{\underline{m}}$ & $r$ & $r$ & \\
\hline & \multirow{2}{*}{ 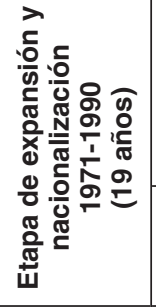 } & $\therefore$ & ட̊ & $\stackrel{m}{\stackrel{m}{N}}$ & $\hat{\hat{\theta}}$ & $\stackrel{N}{N}$ & & & 움 \\
\hline & & $z$ & $\stackrel{9}{\stackrel{9}{2}}$ & $\cong$ & 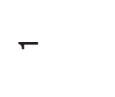 & $r$ & & & $r$ \\
\hline & \multirow{2}{*}{ 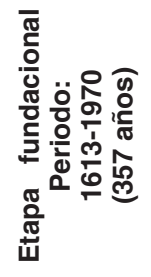 } & $\circ$ & @) & $\begin{array}{l}\infty \\
i^{\infty}\end{array}$ & & & & & \\
\hline & & $z$ & 우 & $\mp$ & & & & & \\
\hline & \multirow{2}{*}{ 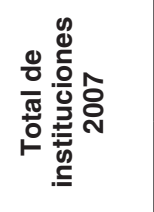 } & ㅇㅇㅡ & $\hat{\hat{m}}$ & $\hat{o}$ & is & $\stackrel{\rho}{\rho}$ & : & : & : \\
\hline & & $z \stackrel{N}{\stackrel{2}{~}}$ & $\stackrel{\infty}{\infty}$ & F & 0 & $\stackrel{\nabla}{ }$ & $\tau$ & $r$ & $r$ \\
\hline & & & 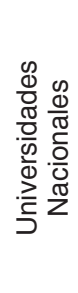 & 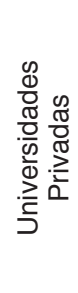 & 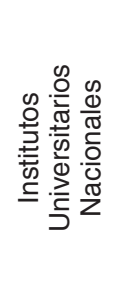 & 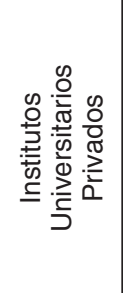 & 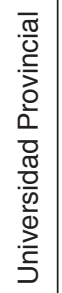 & 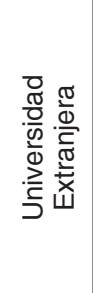 & 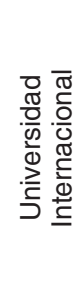 \\
\hline
\end{tabular}

Fuente: http://www.me.gov.ar/spu/Servicios/Autoridades_Universitarias/au listado_de_universidades. htm

Elaboración Propia 
A partir de la década del '50 se produjo la ampliación y masificación de la matrícula post secundaria, la cual se distribuyó en función de la oferta educativa universitaria. Desde entonces, la universidad fue creciendo en sucesivas oleadas. Como consecuencia de la primera expansión de carácter privado, de las 7 universidades públicas que Argentina tenía en 1956, pasó a tener 30 en 1970. Una segunda oleada de creación de instituciones públicas entre $1971 \mathrm{y}$ 1990, dio lugar a la creación de 19 universidades nacionales (incluidas algunas provinciales que fueron nacionalizadas) y 12 universidades privadas en distintas regiones del país. A partir de 1991, comenzó una tercera ola de expansión de carácter mixto con un claro predominio del sector privado.

Cada una de estas etapas representa un periodo significativo en la consolidación de las políticas de expansión de la educación superior y tendencias del cambio promovido por los órganos de gobierno. La denominada Etapa Fundacional (1613-1970) se distingue por la creación de las universidades que cimentaron el sistema universitario protagonista del Movimiento Reformista en la Argentina; la Universidad de Córdoba (creada en 1613), la Universidad de Buenos Aires (creada en 1821) y la Universidad de La Plata (creada en 1905). Posteriormente se fundaron otras 7 universidades nacionales que configuraron el subsistema público universitario. En la segunda etapa aquí nombrada como período de Expansión y Nacionalización de las universidades (1971-1990), se consolidaron además las universidades privadas tradicionales en la Argentina. En la tercera etapa llamada Expansión Privatista (1991-2007) regulada por la Ley Universitaria 24.521 se crearon nuevas universidades públicas y privadas con un modelo organizacional alternativo al de la cultura reformista.

De este modo se fue conformando un entramado institucional altamente heterogéneo y diverso en el que coexisten universidades tradicionales y nuevas, públicas y privadas, católicas y seculares, de élite y masivas, profesionalizantes y de investigación. Sin embargo, las universidades públicas concentran el 83,5\% de la matrícula total de estudiantes y gran parte de ellas realizan el conjunto de misiones que el sistema de educación superior norteamericano tiene asignado a cada tipo de institución diferente. En la actualidad, la oferta universitaria cubre prácticamente todas las regiones del país.

Si bien en el Cuadro 1 queda evidenciada la tendencia a la expansión de las universidades privadas que superan a las públicas, el Cuadro 2 muestra que el porcentaje de nuevos inscriptos en el sector privado en el año 2005, no supera el $21 \%$ y el $79 \%$ de los matriculados se encuentra en las universidades públicas. La tendencia en cuanto a los institutos universitarios es mas pareja ya que el 59\% esta matriculado en las Institutos de gestión estatal y casi el 41 $\%$ en los de gestión privada. 
Cuadro 2

Nuevos matriculados por sector de gestión, 2005

\begin{tabular}{|c|r|r|r|r|r|r|}
\hline \multirow{2}{*}{ Institución } & \multicolumn{2}{|c|}{$\begin{array}{c}\text { Gestión Estatal } \\
2005\end{array}$} & \multicolumn{2}{|c|}{$\begin{array}{c}\text { Gestión Privada } \\
2005\end{array}$} & \multicolumn{2}{l|}{ Total Nuevos Inscriptos } \\
\cline { 2 - 7 } & \multicolumn{1}{|c|}{$\mathrm{N}$} & \multicolumn{1}{c|}{$\%$} & $\mathrm{~N}$ & \multicolumn{1}{c|}{$\%$} & $\mathrm{~N}$ & \multicolumn{1}{c|}{$\%$} \\
\hline Universidades & 289,708 & 80 & 73,265 & 20 & 362,973 & 100 \\
\hline Institutos Universitarios & 4,249 & 59,4 & 2,907 & 40,6 & 7,156 & 100 \\
\hline Total & 293,957 & & 76,172 & & 370,129 & \\
\hline
\end{tabular}

Fuente: SPU, Anuario 2005 de Estadísticas Universitarias, página 173

Elaboración Propia

En cuanto a la expansión del sistema de educación superior en su conjunto, esto es universitario y no universitario, el primero sigue dominando el escenario con un $73,5 \%$ de matriculados respecto del $26,5 \%$ de alumnos en el nivel terciario no universitario. Estas tendencias cuantitativas son altamente significativas cuando las comparamos con Brasil y México que muestran un comportamiento inverso, con universidades públicas que ofertan pos grados académicos para élites y una masiva oferta de instituciones terciarias privadas, para la mayoría de la población.

En el Cuadro 3 se puede observar que la mayor tasa de crecimiento anual de los estudiantes entre 2001 y 2005 , se da en los institutos universitarios privados con un 43,63 aunque estos estudiantes representan el 16,50 \% comparado con los estudiantes de las universidades de gestión estatal y privada que representan el $83,50 \%$ del total como se observa en el Cuadro 4.

Desde el punto de vista del régimen financiero, las universidades nacionales son gratuitas y gozan de autarquía universitaria aunque la Ley de Educación Superior promulgada en 1995 introduce cambios al respecto.

Uno de los propósitos de la creación de nuevas universidades públicas en el cono urbano bonaerense (Universidad de Quilmes -creada en 1989-, Universidad de Tres de Febrero - creada en 1995-, Universidad General Sarmiento -creada en 1992- , Universidad General San Martín creada en 1992-, etc.) fue romper con el modelo reformista de las universidades públicas tradicionales, cambiando criterios clave de funcionamiento. Reemplazaron al tradicional gobierno universitario por un órgano de gestión universitaria comprometido con la obtención de recursos alternativos, el ingreso irrestricto por un ingreso selectivo, la gratuidad por el arancelamiento (en el caso de la Universidad de 
Marcela Mollis
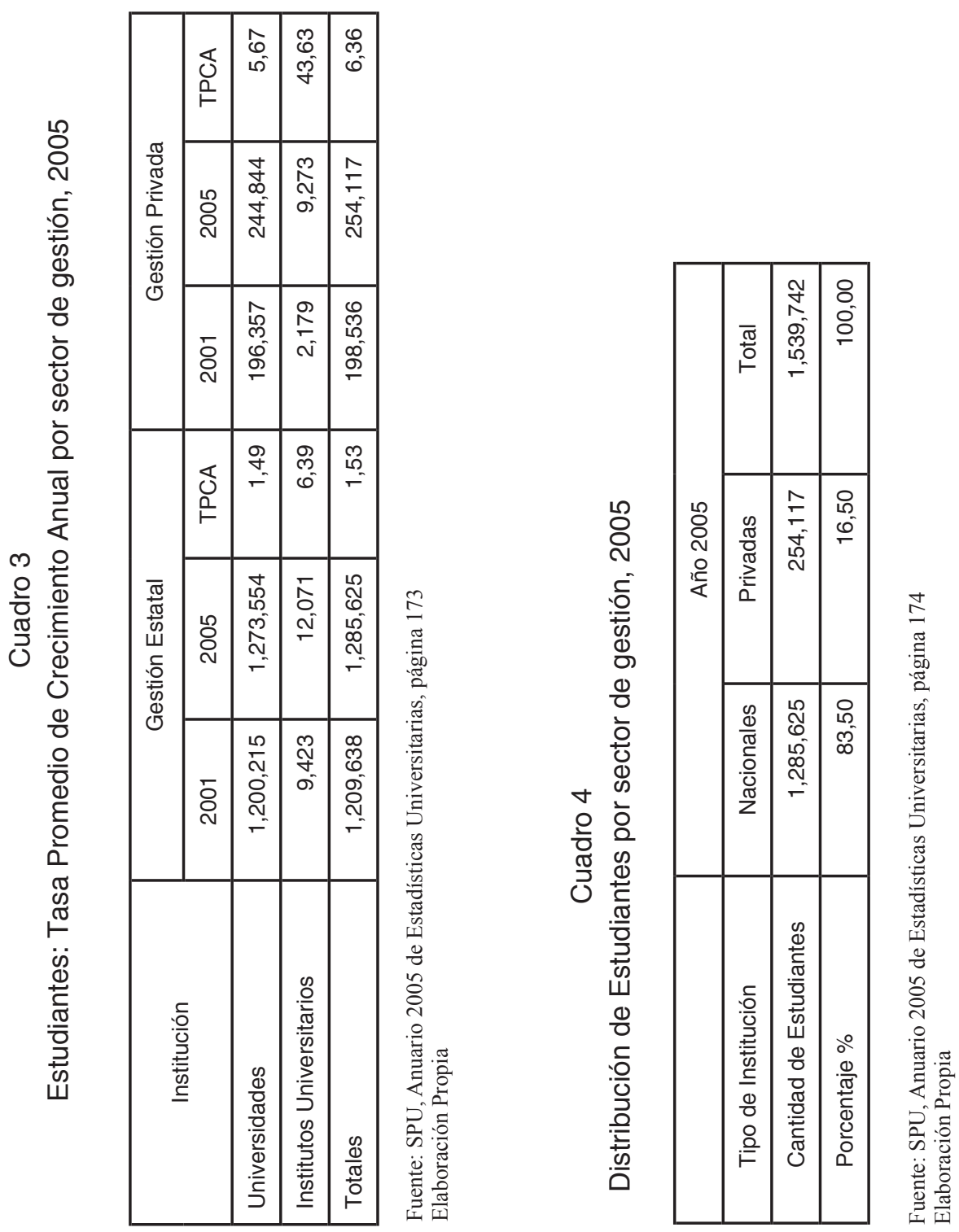

Quilmes), los docentes auxiliares por profesores temporarios por contrato, profesores con dedicación simple por profesores con máxima dedicación a la enseñanza e investigación y responsabilidad tutorial (seguimiento de estudiantes, horarios de oficina para atención de consultas), salarios diferenciados, carreras cortas con salida laboral, diplomas intermedios, carreras a distancia 
y aplicación de tecnologías virtuales, orientación profesionalizante y poca o ninguna oferta en las áreas de ciencias básicas o aplicadas. Si bien estas características están asociadas con los nuevos modelos universitarios, no se presentan en todos los casos del mismo modo.

Lo que se pudo apreciar al término del primer quinquenio de funcionamiento de estas instituciones, es que por su tamaño organizacional, existe una relación más directa entre la gestión de la universidad y el sector académico (el profesor está más “supervisado" por el sector que gestiona o administra la universidad), se produjeron convenios o acuerdos con los municipios o gobiernos locales en las jurisdicciones donde funcionan dichas universidades para ampliar las fuentes de financiamiento y satisfacer necesidades de la comuna, la oferta de pos grados profesionales resultó clave para la obtención alternativa de recursos, hay menor número de estudiantes por profesor en los cursos de grado, y menor porcentaje de deserción estudiantil debido a la incidencia de las pruebas de admisión. Entre las desventajas observadas, podemos mencionar la superposición de oferta de carreras grado en áreas cercanas a otras universidades públicas, con un número de alumnos tan escaso que no justifica la existencia de la oferta, pocos profesores con perfiles de excelencia, y baja inversión en los recursos bibliotecarios (pocos libros y colecciones de revistas científicas). De cualquier modo, medir el impacto de este modelo "modernizador" universitario en la calidad de los nuevos egresados, es todavía una tarea pendiente para poder evaluar las ventajas o desventajas de dichos parámetros modernizadores derivados de una agenda de reforma internacional.

En el período 1982-1992 las universidades nacionales no solo duplicaron su población estudiantil sino también el número de docentes.

\section{Cuadro 5}

Cargos docentes por categoría, 2005

\begin{tabular}{|c|r|r|r|}
\cline { 2 - 4 } \multicolumn{1}{c|}{} & \multicolumn{1}{c|}{ Total } & \multicolumn{1}{c|}{ Profesores $^{*}$} & Docentes Auxiliares $^{* *}$ \\
\hline Universidades Nacionales & 119,339 & 49,041 & 70,298 \\
\hline Porcentaje \% & 100 & 41,1 & 58,9 \\
\hline
\end{tabular}

* Profesores: Titular, Asociado y Adjunto

** Docentes Auxiliares: Jefe de Trabajos Practicos, Ayudante de $1^{\mathrm{a}}$ y $2^{\mathrm{a}}$

Fuente: SPU, Anuario 2005 de Estadísticas Universitarias, página

Elaboración Propia 


\section{Cuadro 6}

Cargos docentes por categoría y dedicación

\begin{tabular}{|c|c|c|c|c|c|c|c|c|}
\cline { 2 - 9 } \multicolumn{1}{c|}{} & \multicolumn{2}{c|}{ Exclusiva } & \multicolumn{2}{c|}{ Semi Exclusiva } & \multicolumn{2}{c|}{ Simple } & \multicolumn{2}{c|}{ Totales } \\
\cline { 2 - 9 } \multicolumn{1}{c|}{} & $\mathrm{N}$ & $\%$ & $\mathrm{~N}$ & $\%$ & $\mathrm{~N}$ & $\%$ & $\mathrm{~N}$ & $\%$ \\
\hline Profesores & 9,886 & $20,2 \%$ & 12,424 & $25,3 \%$ & 26,731 & $54,5 \%$ & 49,041 & $100 \%$ \\
\hline Auxiliares & 5,563 & $8 \%$ & 13,936 & $20 \%$ & 50,798 & $72 \%$ & 70,298 & $100 \%$ \\
\hline
\end{tabular}

Fuente: SPU, Anuario 2005 de Estadísticas Universitarias, página 180 Elaboración Propia

El Cuadro 5 muestra que del total de docentes universitarios en el año $2005,41 \%$ tiene la categoría de Profesor (adjuntos, asociados y titulares) y el $59 \%$ restante pertenece a la categoría de docentes auxiliares. En el Cuadro 6 se puede observar que del $41 \%$ de los profesores de las universidades nacionales, el $20,2 \%$ a su vez, tiene dedicación exclusiva (dedicación exclusiva a las tareas de enseñanza e investigación con 40 horas semanales), el 25,3\% tiene dedicación semi-exclusiva (20 horas semanales) y el 54,5\% tiene dedicación simple (menos de 10 horas semanales). A su vez entre los auxiliares docentes, sólo el $8 \%$ tiene dedicación exclusiva y el $20 \%$ semi exclusiva, en tanto que el $72 \%$ restante tiene dedicación simple. Desde el punto de vista de las estrategias de enseñanza estos indicadores sugieren que la mayor parte del tiempo de clase de nuestros jóvenes universitarios, están en contacto con jóvenes profesionales, sin experiencia profesional ni pedagógica. Las causas de esta deficiente distribución de nombramientos y dedicaciones tiene un fundamento financiero: es más barato contratar muchos jóvenes ad honorem o con salarios bajos para atender una población estudiantil masiva, que contratar profesionales con expertisia, antigüedad y máxima dedicación. Aparece una tendencia en nuestro sistema universitario muy clara: en aquellas unidades académicas donde recae la responsabilidad docente en los profesores más que en los auxiliares, donde hay mayor dedicación horaria y mayor número de docentes con diplomas o certificados de pos grado, se eleva la calidad de la enseñanza de los cursos. Sin embargo la masividad de la matrícula privilegia el reclutamiento de docentes jóvenes sin experiencia. 


\section{Globalización y nuevo cosmopolitismo: la cultura empresarial en la educación superior}

Las tendencias globales de la educación superior en el último par de décadas pueden resumirse en una frase: los sistemas de educación superior están en "transición en casi todo el mundo" (Forest \& Altbach, 2006). La descripción particular que define la transición de un tipo de modelo a otro, se caracteriza por el difundido slogan: del Estado al mercado. El poder económico del norte se expresa en la supremacía de un modelo de educación superior orientado a la satisfacción del mercado laboral global, que no sólo avanza en la región sino en los países post socialistas. Así comienza el último documento sobre educación superior editado conjuntamente por los miembros de la Task Force on Higher Education and Society del Banco Mundial en acuerdo con la UNESCO:

"La economía mundial está cambiando en la medida que el conocimiento reemplaza al capital físico como fuente de riqueza presente (y futura). La tecnología también refleja este proceso a través de la información tecnológica, la biotecnología y otras innovaciones que orientan las notables transformaciones en el modo de vivir y trabajar. A medida que el conocimiento se vuelve más importante, la educación superior también crece en importancia. Los países necesitan educar a la gente joven con estándares más elevados ya que el diploma es un requerimiento básico para cualquier trabajo calificado. La calidad del conocimiento generado por las instituciones de educación superior y su potencial contribución a una economía en gran escala, se vuelve un punto crítico para la competitividad nacional. Esto constituye un serio desafío para el mundo subdesarrollado" (World Bank, 2000:9)

Las visiones de la social democracia están siendo reemplazadas por las políticas orientadas al mercado: es el imperio de la "mercadotecnia" (Marginson S. \& Mollis, M., 2001). El papel de los gobiernos está siendo re inventado, "las nuevas tecnologías han suplantado las percepciones anteriores" y la globalización y el internacionalismo han acelerado la difusión de los nuevos valores de la cultura empresarial extendida a las instituciones sociales y culturales (De Sousa Santos, 2005). El párrafo siguiente ejemplifica los fundamentos globales para que los países del mundo subdesarrollado tomen conciencia del imperativo económico que justifica la transformación de los sistemas de educación superior:

“Actualmente en la mayor parte del mundo subdesarrollado, aunque existen notables excepciones, sólo se lleva a cabo marginalmente el potencial que la educación superior tiene para promover el desarrollo" (World Bank, 2000: 10) 


\subsection{La agenda de la globalización y su impacto en la educación superior argentina}

Un año antes que comience el 2000, con el lamentable suceso de un Ministro de Economía anunciando un recorte de 300 millones de dólares del presupuesto educativo, y la decisión que el sector más castigado por el ajuste acordado con los representantes del Fondo Monetario Internacional, debía ser Educación y no los "gastos reservados del gobierno", se puso en evidencia el reinado de las Cuatro "I" de Kenichi Ohmae. En su obra The End of the Nation-State, Ohmae reconoce que el estado-nación está económicamente esclerótico y que, aunque juegue un papel en la economía mundial, perdió completamente su capacidad de control sobre la economía nacional ante la imposibilidad de controlar el cambio y proteger su moneda. Las cuatro "I" orientadoras del desarrollo financiero y la expansión económica, son para este autor nipón, la Industria, la Información tecnológica, los Inversores y los Individuos como consumidores. "El estadonación se ha vuelto disfuncional como organización económica: carece de incentivos (como históricamente los tuvo en la era del capitalismo industrial), credibilidad, herramientas, legitimidad, debido a que los flujos financieros están condicionados por gente e instituciones sobre quienes los estados-nación no tienen control práctico" (Ohmae, 1995: 12; citado en Torres, 1998: 78).

La respuesta local a la lógica global, está representada por las estadísticas que exhiben el imperativo categórico del modelo neo liberal aplicado en Argentina: la exclusión social ${ }^{1}$. Hacia fines de 1999 , Argentina tenía un $25,9 \%$ de pobres del total de la población (33.000.000 millones).Estos indicadores constituyen la respuesta local a las demandas internacionales a favor del ajuste fiscal. Son el resultado de las políticas de exclusión, y marginación social conjuntamente con la concentración de la riqueza, la privatización de las empresas públicas y las leyes de flexibilización laboral, acuerdos derivados de negociaciones crediticias efectuadas con el Fondo Monetario Internacional. Podemos concluir que en Argentina se ha producido el imperio de las cuatro "I" del autor japonés Ohmae.

El proceso de privatizaciones, constituye uno de los instrumentos fundamentales para el cambio de régimen de acumulación capitalista en Argentina. A partir de ese proceso se modifica, en parte, la intervención del Estado en la

1 La falta de trabajo es sin duda la variable con mayor incidencia en los índices de pobreza y sub ocupación. En Argentina existen 3.500.000 de personas con problemas de empleo: 1,7 millones están desocupados y 1,8 millón se encuentran sub ocupados (los que trabajan menos de cuatro horas diarias aunque quieran hacerlo por más horas). La tasa de desempleo más alta se registró en mayo de 1995 con el 18,4 \% en tanto que la subocupación alcanzó el 11,3\%. Desde entonces la desocupación fue disminuyendo hasta el 12,4\% en octubre de 1998 y la subocupación aumentó al 13,6\% (Ministerio de Economía e INDEC, 1999) 
economía; se abandona la función "reguladora" de las empresas del Estado, porque todas se privatizan (electricidad, teléfonos, compañía aérea, y finalmente los yacimientos de petróleo). Sin embargo y paradojalmente, el Estado es entendido como sinónimo de "gobierno central" y controla las instituciones universitarias a partir de un tipo de función regulatoria que emana de la Ley de Educación Superior promulgada en 1995.

Martin Carnoy (1998) señala que la Economía Política considera a "la educación como un factor condicionado por las relaciones de poder entre los diferentes grupos económicos, sociales y políticos". Para los economistas políticos no se puede analizar por separado el estudio de los sistemas educativos del análisis explícito o implícito de los fines y el funcionamiento del gobierno. Dado que el poder, en parte, se expresa a través del sistema político de una sociedad, cualquier modelo de economía política de las reformas educativas tiene como fundamento una "teoría del Estado". En el caso de las reformas neo liberales, esta teoría ha reemplazado el concepto de Estado por el de Gobierno que administra los asuntos públicos como privados y reconoce en el mercado su natural fuente de legitimidad. Sin lugar a dudas, la llamada crisis del "Estado Benefactor" se encuentra en el centro del debate socio-económico tanto en América Latina como Argentina, cuyas consecuencias se agudizaron a partir de la década del '70, acompañadas por un fuerte endeudamiento externo, por el estancamiento de los índices de crecimiento económico y del empobrecimiento y marginación de grandes sectores de la población con el consecuente ensanchamiento de las desigualdades sociales.

Por estas razones entre otras, consideramos que la crisis que afecta dramáticamente a la educación universitaria en el tercer milenio, es una crisis de identidad. Apelando a la metáfora del médico, se ha elaborado un diagnóstico "global" de una enfermedad equivocada para forzarnos a comprar el remedio bajo el formato de créditos financieros que impactan en las políticas públicas internacionalmente diseñadas. Las reformas impulsadas por el Banco Mundial en América Latina y en los países pos comunistas parten de diagnósticos globales, universales y homogéneos. Los sucesivos informes (World Bank, 2000) se caracterizan por un tipo de discurso economicista, a-histórico, negador de la pluralidad cultural, orientado a la privatización de las universidades públicas y al reconocimiento pragmático del mercado como única fuente de "innovación y calidad". El fomento de las carreras orientadas al sector servicios refuerza el predominio de un perfil empresarial globalizado ${ }^{2}$.

2 Las carreras preferidas por los jóvenes en Argentina y Hungría, son: Administración, Marketing, Informática y Ciencias de la Comunicación. 
Nuestro diagnóstico local respecto de las reformas universitarias de los 90 ‘s en Argentina, es el siguiente:

- $\quad$ se han perjudicado las tradiciones académicas; ha habido un impacto negativo en el fomento y desarrollo del campo cultural y científico, en las artes, las letras, las ciencias sociales, y las ciencias básicas fundamentalmente

- se ha diversificado la oferta institucional universitaria, aunque la oferta disciplinar reproduce en forma homogénea, la oferta de carreras cortas y con salida al sector de servicios, paralelamente se ha disminuído la oferta de las carreras que pertenecen al campo cultural y /o científico

- se ha desalentado - ya sea por falta de financiamiento o por falta de oferta- los pos grados en las áreas científicas básicas y aplicadas y han proliferado los pos grados con orientación profesionalizante

- se ha desprestigiado la universidad pública como institución formadora de la "clase dirigente nacional", orientando las élites hacia algunas universidades nuevas privadas que todavía no tienen tradición para ser reconocidas como de "calidad", y hacia los pos grados en el extranjero (en los Estados Unidos fundamentalmente).

El historiador británico Eric Hobsbawm en una entrevista reciente, asegura que "la caída del Muro del Berlin no sólo significó el triunfo del capitalismo, sino fundamentalmente, la pérdida del único reaseguro por el cual el capitalismo no iba a devorar su propia criatura" (Hobsbawm, 1998). El capitalismo no necesita mostrarse "humanitario" para superar la amenaza del socialismo real que ya no constituye una amenaza. Las dinámicas del capitalismo de mercado -globales y locales- encuentran socios dispuestos a elevar el sacrificio de las personas a cambio de unos indicadores cuantitativos beneficiosos para los inversores internacionales (Slater, 1992).

\section{Tendencias y desafíos de la educación superior en Argentina}

Entre las estrategias que promovió la "nueva agenda de la modernización" en los 90's para conquistar la racionalización financiera, se encuentran: la desregulación y desburocratización administrativa, las privatizaciones, y la reducción de la responsabilidad del Estado central en la prestación de los servicios públicos. La implementación de los procesos de reforma en América Latina es vista por los políticos como un "instrumento para resolver obstáculos" más 
que un fin en sí mismo y por lo general no se contempla el papel clave de los actores educativos involucrados en estos procesos.

Los cuadros que se presentaron mas arriba expresan la diferencia que existe entre la dinámica externa del mercado institucional que representa la tendencia a la privatización tal -como aparece en el Cuadro 1- versus la dinámica representada por las elecciones de los actores - como aparece en el Cuadro 2-

América Latina también exhibe la expansión de la privatización a nivel institucional aunque mantiene la supremacía de la matrícula pública, tal como se desprende de los Cuadros. La elección de los estudiantes latinoamericanos, reproduce su preferencia por los sistemas de educación superior públicos, a pesar de un mercado privado en expansión como consecuencia de la restricción del financiamiento estatal.

Ya fuera por la gratuidad del sistema o por una preferencia del mercado laboral -y fundamentalmente de algunas corporaciones multinacionales (Revista Mercado, 1998:38) que recluta egresados del circuito público de educación superior en las carreras de Administración de Empresas, Ingeniería en Sistemas e Informática, en Argentina la opción dominante sigue estando en el sector público universitario.

Entre las tendencias de las reformas de la educación superior del último decenio en el mundo, reconocemos una común ausencia del Estado de la regulación de los derechos públicos y el fomento de la regulación en aras del interés privado: se privatizan y desregulan la empresas que representan el interés público y se mercantiliza el interés de las clases políticas dirigentes que buscan beneficios privados (Mollis, M. 2006a). Contradictoria fórmula: desregulación y control en ambas esferas con intereses "alterados". El interés privado es defendido por los representantes elegidos por los ciudadanos que gobiernan en nombre del pueblo o de la nación argentina, aunque muchos gobiernan por cuenta de sus ambiciones privadas. Se ha naturalizado la imposibilidad de cambiar, se ha convertido al optimismo pedagógico en el fatalismo de lo posible, en el corset de la esperanza por una comunidad educativa más justa. Hoy, el interés público hacia la educación superior está representado por la elección de los actores, quienes siguen optando mayoritariamente por el sector público educativo. Ya fuera por la gratuidad del sistema de educación superior (universitario y no universitario) ya fuera por la preferencia de ciertas corporaciones del mercado laboral para reclutar egresados de algunas carreras y especialidades de las universidades públicas, los actores dominan el sector público con su presencia mayoritaria. Si bien han crecido y se han desarrollado las instituciones privadas, su matrícula representa un porcentaje menor respecto 
de la matrícula total de la educación superior. En Argentina, contrariamente al caso mexicano y brasileño, la educación pública superior es la opción que recibe mayor demanda todavía. Aunque también hay que reconocer que las clases dirigentes que se formaban en las universidades públicas, hoy completan sus pos grados en las prestigiosas universidades norteamericanas que garantizan su reproducción.

El escenario futuro plantea un importante desafío: la tendencia a la privatización en Argentina, ¿tomará la forma de la privatización del interés de los administradores y gestores de la esfera pública? Algunos funcionarios del sector público defienden los intereses privados que conducen a las políticas académicas opuestas al valor de la excelencia y fomentan el valor del mercado y el clientelismo político. El futuro de la clase política dirigente argentina, de los intelectuales artistas, poetas, filósofos, el futuro de los científicos y de los maestros.¿En manos de quién está? ¿Quién defenderá el interés público de una educación superior orientada al desarrollo del arte, la ciencia, la cultura y la formación cívico-ciudadana?

Estamos en condiciones de reconocer que muchos hacedores de políticas públicas y gestores del destino de las instituciones educativas públicas, tienen "privatizadas" sus mentalidades, u al menos, orientadas a la satisfacción de intereses corporativos ajenos al bienestar general.

\section{Cinco mitos del sistema universitario y una propuesta de cambio}

A pesar de los embates a las universidades públicas nacionales que ya no cuentan con su "socio fundante y fundador de legitimidad pública" el Estadonación que la financia, existe una crisis del modelo institucional universitario que es propia, legítima y justificada en la historia del largo siglo XX. Hoy se cuestiona el concepto de "democracia universitaria" arraigada en el cuerpo de "profesores regulares" (elegidos por concurso) que representan un porcentaje minoritario respecto del total de docentes universitarios. También se reconoce una crisis de representatividad del cuerpo colegiado junto a la crisis del paradigma comunicacional-científico, la crisis de la estructura de facultades del modelo napoleónico, la desvinculación con los otros niveles del sistema, la desconexión con las urgentes necesidades de la sociedad civil, la debilitada voluntad de cambio de sus actores, y por último la pérdida de la identidad comunitaria. La descripción de los mitos que siguen a continuación están vinculados a las crisis del modelo estructural de la universidad pública, la que 
reconocemos como una oportunidad para la refundación de las instituciones portadoras del saber y hacedoras del conocimiento en el siglo XXI.

a. El acceso universal: las universidades públicas masivas de acceso directo, han desarrollado en la última década dispositivos de ingreso que encubren los mecanismos selectivos puestos en juego, ya fueran ciclos iniciales comunes, cursos de verano, cursos tutoriales, etc. Se le suma la composición socio económica del estudiantado, que deja fuera del circuito educativo a los hijos de los obreros o trabajadores de los niveles más bajos

b. Gobierno representativo y democrático: la composición de los claustros está desfasada respecto del significativo número de docentes con las categorías inferiores (“docentes auxiliares") que no pertenecen al cuerpo profesoral; sumado a la no sustanciación de los concursos que lleva a un mínimo porcentaje de profesores como electores y candidatos;

c. Lugar de producción del conocimiento de punta: el feroz desfinanciamiento estatal durante la última década sumado a la baja inversión de Producto Bruto en Investigación y Desarrollo de los países periféricos, desalienta, entorpece y anula las posibilidades de construcción de pensamiento científico empírico, puro y de punta en las universidades públicas

d. Meritocracia para acceder a los cargos por concurso: los concursos docentes en la última década han devenido en dispositivos políticoclientelares más que académicos; jurados observados, dictámenes recusados y presentaciones judiciales dan cuenta de la arbitrariedad de los mecanismos instalados

e. La comunidad universitaria está quebrada; en lugar de una comunidad existe un conjunto de identidades superpuestas y enfrentadas de actores gremiales, sujetos ultraindividualistas y jóvenes no tan jóvenes desencantados. Todos tienen en común la ausencia de pertenencia institucional que los estimule a la innovación.

Las universidades públicas argentinas y latinoamericanas, fascinadas por la ilusión de una identidad homogénea global, han desnaturalizado sus históricas funciones sociales. La comunidad académica heredera de la tradición comunitaria medieval, se ha desvanecido ante el ultraindividualismo profesoral. La heterogeneidad del cuerpo de profesores universitarios, se expresa en una gama que va desde el profesor investigador incentivado (que representa el 18\% de la 
población nacional de profesores universitarios en Argentina) hasta el enseñante recién egresado (que representa una mayoría significativa de la población docente universitaria). La identidad de los profesores de las universidades públicas se encuentra en tránsito del académico al consultor internacional debido a que "prestigio y honorarios" provienen de otras fuentes de financiamiento como las agencias bancarias (nacionales o internacionales) o el gobierno central.

Por lo dicho hasta aquí, estamos en condiciones de asegurar que las universidades públicas frente a las transformaciones promovidas por la globalización, enfrentan el mayor desafío desde su etapa fundacional: la supervivencia.

Para sobrevivir y recrear el sentido fundacional, es necesario acordar una descripción de las urgentes prioridades. ¿Por dónde comenzar? Habrá que reconocerse para cambiar. Reconocer el déficit pedagógico de los planes de estudio para formar profesores que enseñan en los otros niveles del sistema educativo. Reconocer la necesidad de reconstruir la o las misiones institucionales y el valor del conocimiento. Reconocer la crisis de representatividad de los cuerpos colegiados y la disfuncionalidad de la estructura administrativa. Proyectar la investigación hacia la satisfacción de las urgencias culturales y sociales locales, promover la formación de lideres políticos con sensibilidad pública y una ética social. Por último, habrá que pedagogizar los problemas de la universidad, politizarlos, despartidizarlos, habrá que filosofar sobre el sentido, la misión y la praxis universitaria para conquistar un proyecto soberano sustentado en una epistemología social del conocimiento local.

\section{Bibliografía}

Carnoy, Martin (1998) “The Political Economy of Education”, in: Kempner, K, Mollis, M, Tierney, W. (editors) (1998) Comparative Education: ASHE READER, Simon \& Schuster Custom Publishers, MA, USA

De Sousa Santos, B. (2005) La Universidad en el Siglo XXI, Laboratorio de Políticas Públicas (LPP) y Miño y Dávila, Buenos Aires

Dirie, C, Mollis, M. y otros (2001) Mapa de la Oferta de la Educación Superior en Argentina, Documento preparado para la Comisión Nacional de Mejoramiento de la Calidad Universitaria, Ministerio de Cultura y Educación de la Argentina (Versión PDF) 
Dono Rubio, S, (2001) Diversificación institucional en el contexto de las reformas de la educación superior: los colegios universitarios, adaptación al mercado? Ponencia presentada a la Conferencia Internacional: Globalisation And Higher Education: Views From The South, University of the Western Cape, Cape Town, South Africa, March 27-29 (Informe parcial del proyecto de investigación UBACYT dirigido por Marcela MOLLIS titulado: Las Reformas De La Educación Superior en Contextos Institucionales Diferentes: Los Casos De Argentina y Finlandia)

Hobsbawm, Eric (1998) "El Capitalismo perdió todo miedo", Entrevista; Diario El Clarín, Buenos Aires, Noviembre 22, p 4-9.

Marginson, S. \& Mollis, M. (2001) “The door opens and the Tiger leaps. Theories and Reflexivities of Comparative Education for a Global Millennium", en: Comparative Education Review, University of Chicago Press, Vol. 45, No 4, Nov. pp. 581-615

Mollis, Marcela (2000) "The Americanisation of the Reformed University in Argentina", in: Australian Universities Review, Published by NTEU, Vol. 42, No 2 \& Vol 43, No 1, pp. 45-52

Mollis, M. (2001 a) La Universidad Argentina en Transito, Fondo de Cultura Economica, Buenos Aires, México

Mollis, M. (2006a) "Latin American University Transformation of the 90's: Altered Identities", en: Forest, J.\& Altbach, Ph. (Editors) 2006 International Handbook of Education, Vol. 18, Springer Publisher, Berlin, Heidelberg, New York

Mollis, M. (2006b) “Geopolítica del saber: biografías recientes de las universidades latinoamericanas" en: Teichler, Ulrich editor (2006), Reformas de los modelos de la educación Superior en Europa, Japón y América Latina: Análisis Comparados, Vol. 2. Serie Educación Superior, Educación Comparada y Trabajo, Universidad de Kassel y Facultad de Filosofía y Letras, UBA. Miño y Dávila Editores, Consejo Editor Facultad de Filosofía y Letras, Buenos Aires, Argentina

Revista Mercado, (1998) "Qué egresados prefieren las top 10 del mercado?", Oct-Dic, Buenos Aires, pp. 36-40

Slater, David (1992) "Poder Y Resistencia en la Periferia. Replanteando algunos temas críticos para los años 90”, en: Nueva Sociedad, No 122, Nov- Dic, Caracas, Venezuela, pp. 35-46 
SPU-MEC (2005) Anuario 2005 de Estadísticas Universitarias, Documento PDF

Torres, Carlos. (1998) Democracy, Education and Multiculturalism. Dilemmas of Citizenship in a Global World, Rowman \& Littlefield Publishers, Inc, USA, pp. 77

World Bank (2000) Higher Education in Developing Countries, Peril and Perish, WB and the Task Force, Ford Foundation, Washington DC 